PHYSICAL REVIEW MATERIALS 4, 082602(R) (2020)

Rapid Communications

\title{
Quantitatively consistent scale-spanning model for same-material tribocharging
}

\author{
Galien Grosjean (1), Sebastian Wald, Juan Carlos Sobarzo, and Scott Waitukaitis \\ IST Austria, Lab Building West, Am Campus 1, 3400 Klosterneuburg, Austria
}

(Received 12 June 2020; revised 6 July 2020; accepted 31 July 2020; published 17 August 2020)

\begin{abstract}
By rigorously accounting for mesoscale spatial correlations in donor/acceptor surface properties, we develop a scale-spanning model for same-material tribocharging. We find that mesoscale correlations affect not only the magnitude of charge transfer but also the fluctuations-suppressing otherwise overwhelming charge-transfer variability that is not observed experimentally. We furthermore propose a generic theoretical mechanism by which the mesoscale features might emerge, which is qualitatively consistent with other proposals in the literature.
\end{abstract}

DOI: 10.1103/PhysRevMaterials.4.082602

Tribocharging, i.e., charge transfer between materials during contact $[1,2]$, plays a critical role in natural phenomena [3-7], industrial processes [8,9], and energy harvesting devices [10-12], yet resists interpretation. One fundamental roadblock has been the inability to identify the atomic-scale mechanism, and in particular the charge carriers, i.e., ions vs electrons [2]. An equally important roadblock is the lack of quantitative agreement between experiments and theory. Experimentally, issues such as the difficulty of measuring contact areas render much data qualitative (e.g., the sign or scale of charging) [13-18]. Theoretical advances are stymied by the multiscale nature of the effect, where one must simultaneously account for probabilistic effects at the atomic scale $(<1 \mathrm{~nm})$, unexplained emergent features at the mesoscale $(\sim 1 \mu \mathrm{m})$, and then through these explain the familiar behavior of the macroscale ( $>1 \mathrm{~mm})$.

Same-material tribocharging, where charge is exchanged between identical materials, is perhaps the most puzzling manifestation of the phenomenon. It has been attributed to trapped electrons [19-25], induced polarization [26-30], or mechanochemistry [8,31-33]. Yet, experiments on samematerial tribocharging have produced some of the most valuable clues. Using soft (Young's modulus $\sim 1 \mathrm{MPa}$ ), atomically smooth (roughness $<1 \mathrm{~nm}$ ) polymers to achieve conformal contact, Apodaca et al. found that the magnitude of charge transfer grows with the square root of the contacting area [34]. They proposed that the surface consists of equally-sized, randomly-assigned donor/acceptor sites [Fig. 1(a)], each capable of giving/receiving one unit charge. This allowed them to recover $\overline{|\Delta Q|}=C \sqrt{A}$, where the prefactor $C$ depends on the length scale of a single site $l_{0}$. As pointed out by Sow et al. [31], $l_{0}$ should be on the scale of one atom, yet fits to

\footnotetext{
*galienmariep.grosjean@ist.ac.at
}

Published by the American Physical Society under the terms of the Creative Commons Attribution 4.0 International license. Further distribution of this work must maintain attribution to the author(s) and the published article's title, journal citation, and DOI.
Apodaca's model required a value of $\sim 0.005 \AA$-more than 100 times smaller than the Bohr radius of hydrogen.

Nonetheless, features suggestive of their idea have been observed but at significantly larger scales. Using Kelvin force probe microscopy on the same polymers, Baytekin et al. found 'mosaics' - regions of neighboring positive/negative charge after contact - that were spatially correlated over scales up to $\sim 450 \mathrm{~nm}$ [35]. Invoking a scaling argument to resolve the site-size inconsistency, Sow independently proposed that mesoscale donor/acceptor regions might exist, though they did not specifically connect these to Baytekin [31]. In this work, we use analysis validated by numerical simulations to thoroughly develop the model of Apodaca in light of the scaling proposed by Sow and the mosaics observed by Baytekin. We obtain an exact version of Sow's scaling argument, which we validate using Apodaca's charging data and Baytekin's mesoscopic length scale. Going further, we investigate the fluctuations in charging, which we find overwhelm any trends in sequential contact charging between two surfaces unless mesoscale donor/acceptor correlations are present. Finally, we propose a general theoretical formulation for how donor/acceptor regions might form, which produces a tunable mesoscopic length scale that leads to charging consistent with our analysis.

We start by explaining a first set of numerical simulations, where we mimic charge transfer between 'synthetic' surfaces (in contrast to physically derived surfaces later) by creating two $N$-element matrices involving three length scales: $l_{0}$, $l$, and $L$. The smallest, $l_{0}$, corresponds to the elementary donors/acceptors of the atomic scale and is represented by a single matrix element. The largest, $L$, corresponds to the macroscopic system size. We assume that there is a single intermediate scale, $l$, that characterizes the mesoscopic correlations observed by Baytekin. (Note they measured charge, but this implies donor/acceptor correlations.) Each matrix element is assigned as donor or acceptor, with probabilities $p$ and $1-p$, respectively. We account for correlations in assignments via thresholding a random scalar field (see Supplemental Material [36]). To perform a 'contact,' we first generate a 'left' and 'right' surface from identical input length 
(a)
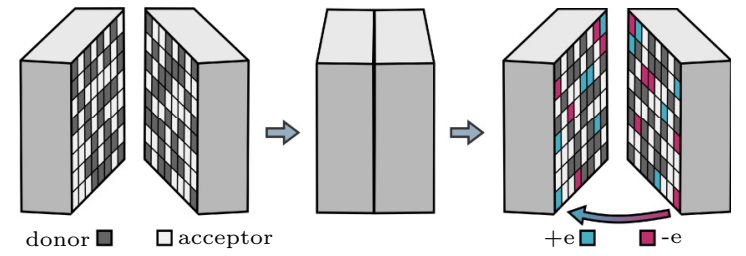

(b)

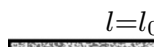

(c)

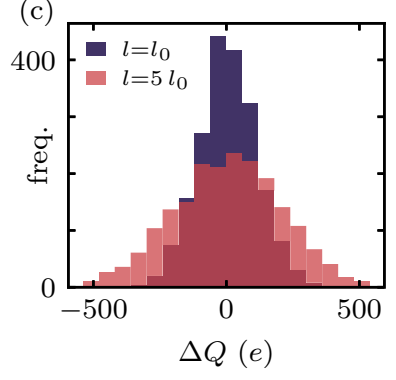

(d)

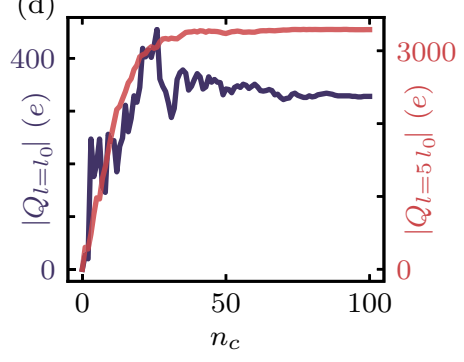

FIG. 1. (a) Apodaca et al. suggested that same-material tribocharging may arise from equally-sized, randomly-distributed donor/acceptor sites, where unit charges $e$ are transferred during contact [34]. (b) Surfaces with identical macroscopic size $L$, microscopic scale $l_{0}$, but different mesoscale correlation lengths $l$. On the left $l=l_{0}$, and on the right $l=5 l_{0}$ (where $l_{0}$ is the site size). (c) The single-contact distribution in $\Delta Q$ is broader when $l=5 l_{0}$ (violet, $\sim 200 e$ ) than when $l=l_{0}$ (pink, $\sim 100 e$ ). (d) For sequential contacts, charge transfer is enhanced when $l=5 l_{0}$, and fluctuations that dominate the $l=l_{0}$ case are suppressed.

scales and probabilities [Fig. 1(a)]. Charge transfer of one unit, $e$, between matrix elements $[i, j]$ occurs if $(1)[i, j]$ on the left/right is a donor, (2) $[i, j]$ on the right/left is an acceptor, (3) the value of an independent random uniform variable is less than the transfer probability $\alpha$, and (4) for sequential contacts, transfer at $[i, j]$ hasn't yet occurred. The net charge transferred is the difference between left-to-right ('right') and right-to-left ('left') transfers.

Figure 1(b) shows two representative surfaces, one with $l=l_{0}$ and the second $l=5 l_{0}$. Although their only difference involves the length scale $l$, we see stark changes in the charging behavior when we contact two surfaces with $l=l_{0}$ vs two with $l=5 l_{0}$. In Fig. 1(c), we plot distributions of the charge transferred in the first contact, $\Delta Q$, for 1000 pair instances. As we do not assume any post-contact discharge [37], both distributions are Gaussian and centered at zero, but while $l=l_{0}$ produces a width of $\sim 100 e$, the $l=5 l_{0}$ width is $\sim 200 e$. Correlations also change the behavior during sequential contacts. Figure 1(d) shows two examples of the accumulated charge $|Q|$ vs the number of contacts $n_{c}$ for two different surfaces, each from a different surface pair. (Note either curve from this plot is the same as what is shown in Apodaca Fig. 1(b), although we do not show the
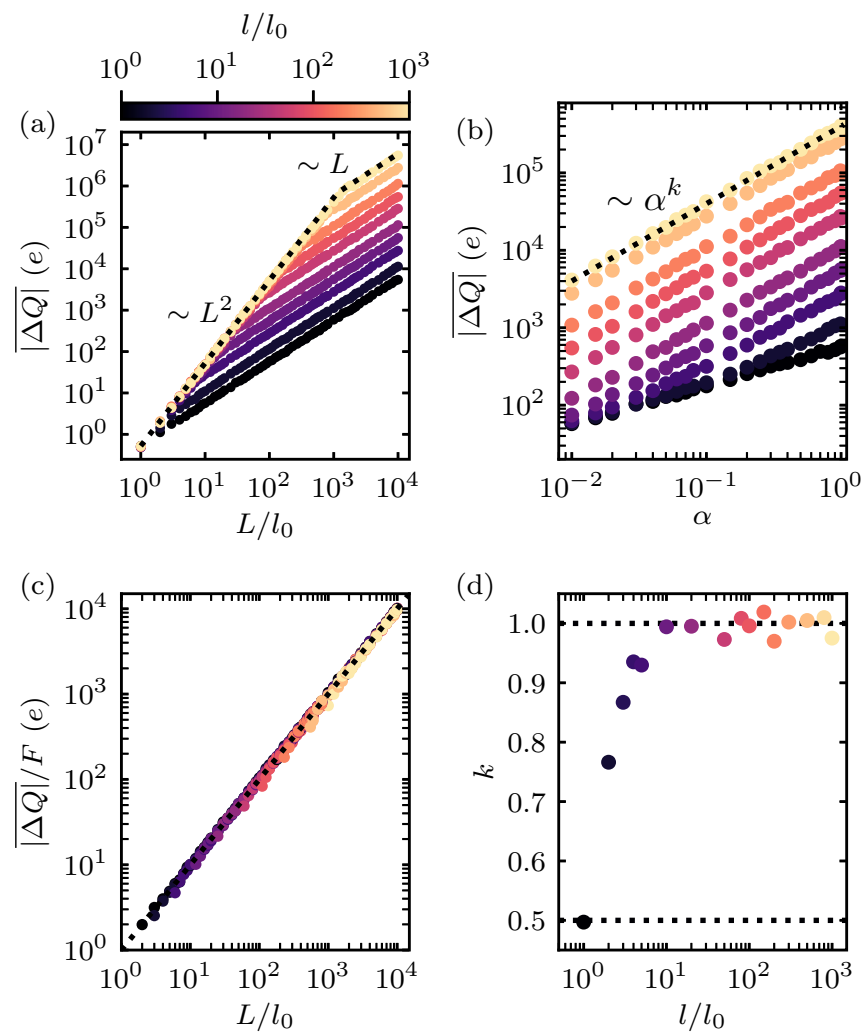

FIG. 2. (a) Average charge transfer $\overline{|\Delta Q|}$ after one contact, for different values of $l$ and $L(\alpha=1, p=0.5)$. Assuming $l<L$, the scaling $\overline{|\Delta Q|} \propto L$ is recovered but with a growing prefactor. (b) For fixed $L$ and $l, \overline{|\Delta Q|} \propto \alpha^{k}$, where $k$ changes with $l$. (c) For $l_{0} \ll l<L$, the data from (a) collapses to a line of slope unity when rescaled using Eq. (2). (d) The exponent $k$ vs $l / l_{0}$. When $l \approx l_{0}$, transfer is probabilistic [Eq. (1)] and $\overline{|\Delta Q|} \propto \sqrt{\alpha}$. When $l \gg l_{0}$, transfer occurs at a fixed rate [Eq. (2)] where $\overline{|\Delta Q|} \propto \alpha$.

negatively charging counterparts as this should be clear from charge conservation [34].) Like the initial transfer, the final charge $Q_{f}$ is typically larger when $l=5 l_{0}$. Additionally, the fluctuations on approach to $Q_{f}$ are significantly different for the two situations [Fig. 1(d)]. When $l=5 l_{0}$ these are hardly discernible, leading to smooth and monotonically increasing behavior (consistent with experiments [34]). However, $l=l_{0}$ they are on the order of $Q_{f}$, leading to nonmonotonic behavior that is not observed experimentally.

We now examine the first contact behavior of these simulations in detail. Figure 2(a) shows the average absolute value of charge exchanged, $\overline{|\Delta Q|}$, for increasing system size and several values of $l$. The scaling $\overline{|\Delta Q|} \propto \sqrt{A} \propto L / l_{0}$ is recovered, but the prefactor steadily increases with $l>l_{0}$. This scaling is limited by the condition that $l<L$. If the length scale $l$ is larger than the system, the scaling becomes $\overline{|\Delta Q|} \propto$ $A \propto L^{2} / l_{0}^{2}$. Figure 2(b) shows that the dependence on the transfer probability $\alpha$ exhibits unexpected nonlinear behavior as a function of the correlation length $l$. At every value of $l$, we see a trend consistent with a power law, i.e., $\overline{|\Delta Q|} \propto \alpha^{k}$. However, the exponent $k$ increases with $l$, starting at $k=0.5$ for $l=l_{0}$ and saturating at $k=1.0$ for $l \gg l_{0}$ [Fig. 2(d)].

To analytically explain these observations, we first consider the case $L \gg l=l_{0}$, here sketching our argument 
(details are in the Supplemental Material [36]). We momentarily focus on right transfers, which occur with compound probability $p(1-p) \alpha$. Absent correlations, all sites $[i, j]$ are independent, hence the total right charge transfer is Gaussian with mean $e N p(1-p) \alpha$ and width $e \sqrt{N p(1-p) \alpha(1-p(1-p) \alpha)}$. A similar distribution exists for left transfer, but technically only when considered independently—simultaneous left/right transfer cannot occur. Nonetheless, the probability for this is small, and we therefore approximate the left/right distributions as independent. The net transfer $\Delta Q$ is thus also Gaussian distributed, with zero mean and width $\sigma=e \sqrt{2 N p(1-p) \alpha(1-p(1-p) \alpha)}$. Neglecting terms like $(p(1-p) \alpha)^{2}$ and considering $\overline{|\Delta Q|}=$ $\sqrt{2 / \pi} \sigma[36]$, we find

$$
\overline{|\Delta Q|}=\sqrt{\frac{2}{\pi}} \frac{e L}{l_{0}} \sqrt{2 p(1-p) \alpha} .
$$

This recovers the $\sqrt{A}$ scaling found in Ref. [34], with the difference that the $\alpha$ dependence is square root rather than linear. This is supported by our simulations in Fig. 2(d). In the Supplemental Material [36], we verify that Eq. (1) collapses our simulated data for wide ranges of $p$ and $\alpha$.

Next we consider the case $L \gg l \gg l_{0}$ (again with details in the Supplemental Material [36]). This fundamentally alters the argument above as the site identities exhibit spatial correlations. To handle this, we first imagine rescaling the system by $l / l_{0}$, leading to surfaces with $N^{\prime}=N /\left(l / l_{0}\right)^{2}$ larger 'patches,' each consisting of many sites. Identities of entire patches still occur with probabilities $p$ and $1-p$. Next, we rescale back to deal with transfers, which still occur independently for each site. During contact, regions of donors face acceptors with the characteristic size of a patch. If the number of sites in these regions $\left(n=\left(l / l_{0}\right)^{2}\right)$ is large, the mean transfer per patch $(\alpha n)$ effectively hides the fluctuations $(\sqrt{n \alpha(1-\alpha)})$-hence we treat $\alpha$ as a rate. Thus, when $l \gg l_{0}$ we have

$$
\overline{|\Delta Q|}=\sqrt{\frac{2}{\pi}} \frac{e \alpha L l}{l_{0}^{2}} \sqrt{2 p(1-p)} .
$$

Here, like in the Apodaca work, the $\alpha$ dependence is linear. The critical difference, however, is the dependence on the intermediate length scale, which amplifies the charge transfer by the factor $l / l_{0}$. We confirm this with our simulated data in Fig. 2(c), where the prefactor $F=\alpha l / l_{0} \sqrt{4 p(1-p) / \pi}$ collapses $\overline{|\Delta Q|}$ when $l \gg l_{0}$ and $l<L$. Qualitatively, the explanation for this amplification is that the variability (i.e., standard deviation) in the number of donors/acceptors on a surface increases with the scale of spatial correlations. One can quickly grasp why by considering the extreme case $l>L$, where each surface is essentially purely donor or acceptor, and consequently $\overline{|\Delta Q|} \propto \alpha e N \propto A$. Indeed, all of the points where $l>L$ follow this scaling in Fig. 2(a). This highlights that same- and different-material tribocharging can be considered two manifestations of a similar underlying phenomenon, only appearing different depending on the scale at which one looks.

We now turn to sequential contacts. As the simulation results of Fig. 3(a) show, repeated contacts with the same surfaces leads to curves in accumulated charge, $|Q|$ vs $n_{c}$, that level off at some value $Q_{f}$. In the Supplemental Material,
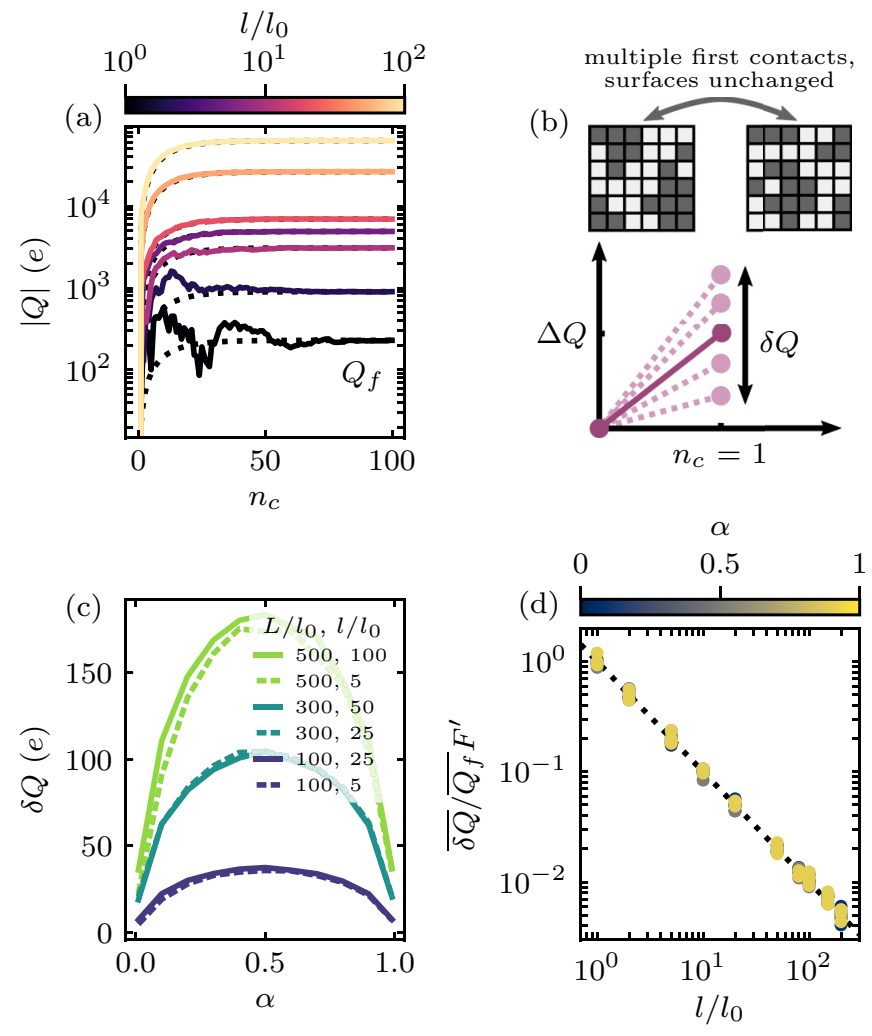

FIG. 3. (a) Sequential contacts lead to accumulated charge, $|Q|$, which ultimately saturates at $Q_{f}$ (here for several $l / l_{0}, \alpha=0.1$ and $p=0.5$ ). For small $l / l_{0}$, fluctuations are on the order of $Q_{f}$, whereas for large $l$ they are suppressed. Dotted lines correspond to Eq. (3) using the measured $Q_{f}$. (b) We quantify fluctuations by repeating the first contact between the same two surfaces and measuring the spread $\delta Q$. (c) For a particular surface pair, $\delta Q$ depends on both $\alpha$ and $L$, but is largely independent of $l$. (d) Ensemble averages of relative fluctuations $\overline{\delta Q} / \overline{Q_{f}}$ collapsed by factor $F^{\prime}=\sqrt{\alpha(1-\alpha) \pi / 2}$ vs $l / l_{0}$, which shows fluctuations are suppressed by spatial correlations. Each point corresponds to the ensemble average over 20 pairs of surfaces of the fluctuations over 50 contacts.

we show that the underlying trend is given by a saturated exponential, i.e.,

$$
Q\left(n_{c}\right)=Q_{f}\left(1-\exp \left(-\alpha n_{c}\right)\right) .
$$

Figure 3(a) also illustrates the presence of fluctuations on approach to $Q_{f}$. For large $l / l_{0}$, these aren't noticeable, but for small $l / l_{0}$ they are overwhelming, and cannot be suppressed even when we increase the system size $L$. We quantify their scale by repeatedly performing first contacts between individual surface pairs ('resetting' each time) and measuring the standard deviation $\delta Q$ [Fig. 3(b)]. In Fig. 3(c) we show $\delta Q$ for a few pairs, which reveals that the fluctuations grow with the macroscale length $L$ and depend strongly on $\alpha$ but are independent of the mesoscale $l$. To analyze why, we note that a particular pair has a fixed donor/acceptor arrangement, which means $\delta Q$ arises solely from $\alpha$. Denoting the number of donors on the left/right that face acceptors on the right/left as $N_{\rightleftarrows}$, one finds that $\delta Q=e \sqrt{\left(N_{\leftarrow}+N_{\rightarrow}\right) \alpha(1-\alpha)}$. In the Supplemental Material [36], we justify using the averages 
$\overline{N_{\rightleftarrows}}=\left(L / l_{0}\right)^{2} p(1-p)$ to find the ensemble expression,

$$
\overline{\delta Q}=e \frac{L}{l_{0}} \sqrt{2 p(1-p) \alpha(1-\alpha)} .
$$

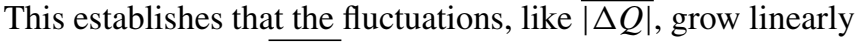
with $L$, but unlike $\overline{|\Delta Q|}$ are independent of $l$. Consequently, they cannot be suppressed by increasing system size, but can be suppressed by the introduction of the intermediate scale $l$. In Fig. 3(d), we collapse the simulated $\overline{\delta Q}$ data to our predicted line with the appropriate rescaling. Our considerations here also have the implication that the fluctuations in charge transferred between a single surface pair should be smaller than the fluctuations between an ensemble of surface pairs, which is consistent with previous observations [17,34].

The last question we posed remains: Why do the intermediate scale correlations emerge in the first place? Several mechanisms have been proposed. Among the most prominent, Sow suggested a mechanochemical mechanism based on statistical fluctuations in localized strain [31]. For inelastic materials at very large strains (i.e., deep into the plastic regime) it has been shown that voids form, which are suggestive [38]. In this case, the process for a growing length scale is clearthe materials are ripped apart-yet the polymers used by Apodaca/Baytekin are highly elastic and not intentionally stretched. There is some indication that uniform in-plane strain during compression could play a role [31], but this does not explain statistical fluctuations in localized strain at a particular mesoscopic length scale. A second, widely held view makes connections to 'islands' of adsorbed surface water $[17,18,37,39,40]$, which definitively do form on surfaces with a similar size scale to charge mosaics [41]. To add to this idea, Burgo et al. showed that water causes virtually all materials it touches to charge negatively [42]—hence patches of 'wet' surface vs 'dry' surface might be interpreted as donor/acceptor regions. Finally, other authors propose that the such heterogeneous features should arise naturally in polymers, pointing to Flory-Huggins theory [43].

We take a more theoretical approach and try to identify generic physical ingredients that could cause a particular length scale to emerge. We propose that a surface formation process which energetically favors neighboring donors (or equivalently, neighboring acceptors) is a viable candidate. We support this idea by developing a second, distinct set of simulations to mimic the physics of surface formation, which are time dependent and latticed based, and which again produce $L / l_{0} \times L / l_{0}$ donor/acceptor matrices. At each time step, we assume a donor site can transition into an acceptor site, and vice versa. The transition probabilities of a given site depend on its neighbors, i.e.,

$$
\begin{aligned}
& P_{A}(v)=P_{0} \exp (-K v) \\
& P_{D}(v)=P_{0} \exp (-K(4-v)),
\end{aligned}
$$

where $v$ is the number of neighbors that are donors (i.e., $v \in$ $[0,4])$. The exponential form is motivated from an Arrheniuslike process where each neighbor modifies a local energy barrier by $\epsilon / k T=K$. This process could equally represent interactions between individual atoms/molecules or processes taking place a continuous medium [44-46], and thus shares qualitative similarities with either water islands or phase (a)
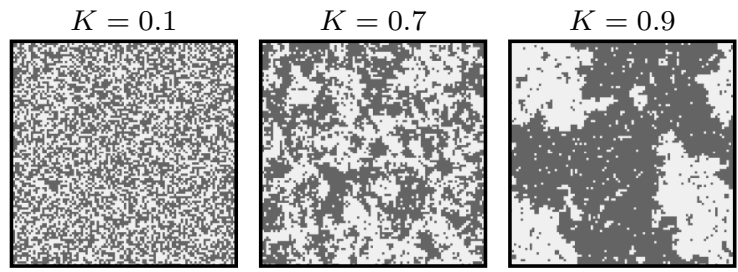

(b)
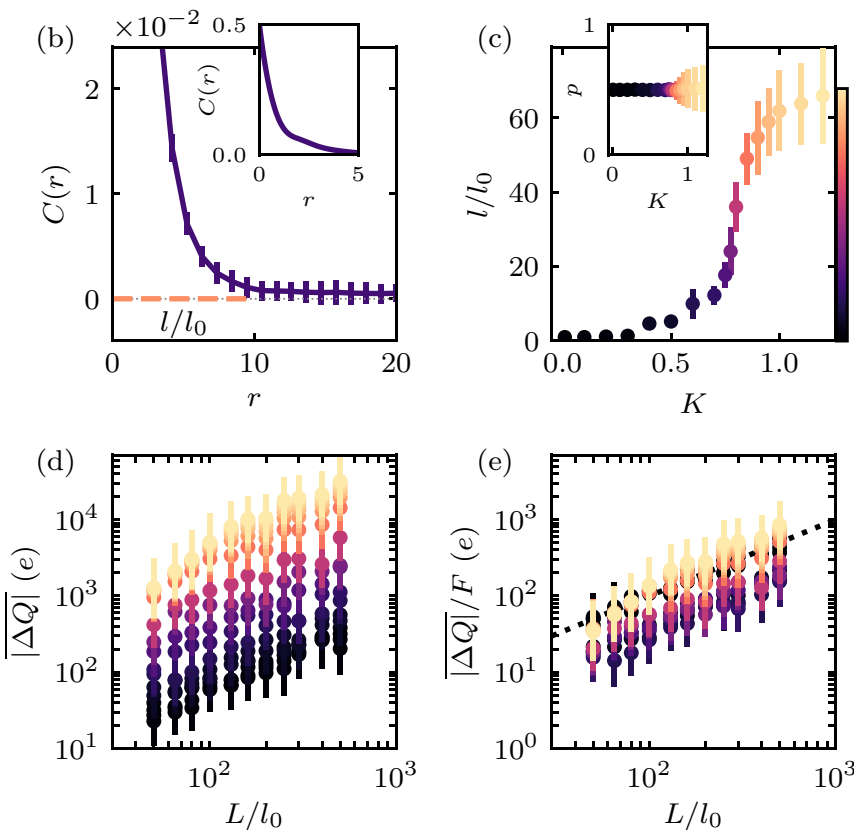

FIG. 4. (a) A generic nucleation process can lead to donor/acceptor regions with a characteristic size $l>l_{0}$. (b) We measure $l / l_{0}$ using the radial correlation function $C(r)$ [Eq. (6)], averaged over several surfaces (error bars represent the standard deviation). The inset shows the whole vertical range. (c) We vary $l / l_{0}$ through parameter $K$ in Eq. (5). The error is calculated from the error on $C(r)$. We keep the same color scale for $l / l_{0}$ in all subfigures. The inset shows that $p$ remains constant. (d) As before, the charge transferred after a contact is amplified by the introduction of $l$. The error bars indicate the standard deviation. (e) We verify that Eq. (2) is still valid. The dotted line is the identity line. Deviations at intermediate $K$ are due to the presence of a spectrum of feature sizes (see Supplemental Material [36]).

separation during curing (i.e., Flory-Huggins). Full details can be found in the Supplemental Material [36].

Starting with an initial arrangement, a surface evolves until it reaches a dynamic equilibrium set by the parameters in Eq. (5) (for a movie, see the Supplemental Material [36]). Three examples for different $K$ are shown in Fig. 4(a). To characterize these surfaces in the context of our analysis, we measure $p$ and $l / l_{0}$. Determining $p$ is trivial. To get $l / l_{0}$ we start by calculating the correlation function,

$$
C(r)=\langle s(R) s(R+r)\rangle-\langle s(R)\rangle\langle s(R+r)\rangle,
$$

where $s$ is the site identity at a position $R, r$ is the distance from the point $R$, and averages denoted by \langle\rangle are over all sites separated by $r$. Figure 4(b) shows an example of $C(r)$ for a particular surface. We use the first zero crossing [within the standard deviation of $C(r)$ ] to define the correlation length 
$l$ as it corresponds to the typical distance before having an equal probability of switching from donor to acceptor (or vice versa). In the Supplemental Material, we show that this identically recovers the input correlation lengths of our other set of simulations [36]. In Fig. 4(c), we show how sweeping through the parameter $K$ ( $P_{0}$ fixed) allows us to explore nearly two orders of magnitude in $l / l_{0}$ (with $p \approx 0.5$ ).

After they have reached a dynamic equilibrium, we freeze these surfaces and then use them as input for contact experiments, with the same transfer rules as before. We generate 20 surfaces for various combinations of correlation length $l / l_{0}$ and system size $L / l_{0}$, and calculate $\overline{|\Delta Q|}$ for every permutation [Fig. 4(d)]. The effect of spatial correlations in this physically-derived system is the same as with the synthetic surfaces, i.e., the magnitude of transfer increases with the correlation length. Figure 4(e) presents the results rescaled using Eq. (2), which largely collapses them onto a single line with the predicted unity slope. In the Supplemental Material [36], we show that the deviation for intermediate $K$ is due a broader 'spectrum' of length scales for $l$ than in our synthetic simulations.

We have shown that the intermediate scale corresponding to donor/acceptor spatial correlations $l$ plays a crucial role in Apodaca's framework for same-material tribocharging. In a single contact, it amplifies the amount of charge transferred, in line with the scaling argument proposed by Sow. In sequential contacts, it suppresses otherwise overwhelming fluctuations, yielding the smooth curves that are observed experimentally. We have furthermore introduced a generic theoretical mechanism for how the intermediate length scale might emerge, which is based on the energetics of donor/acceptor interactions during the formation process. Our work may have implications regarding the carrier and mechanism. If experiments can validate our modification to the Apodaca's framework, this would suggest that the model is quantitative. For example, we predict that single-pair charging fluctuations $(\delta Q)$ should be related to the ensemble fluctuations $(\propto \overline{|\Delta Q|})$ through the correlation length $l$. Measurements of $l$ are already possible with KPFM, and measurements of charge may become precise enough to resolve $\delta Q$ [17]. If such aspects of the model can be validated, then confident extractions of $\alpha$ from experimental data can be made, giving information related to underlying atomic-scale mechanism [47]. Although our model is restricted to surfaces where the macroscopic contact area is known, these considerations become even more important in systems where roughness or stiffness play a role.

We would like to thank Philip Born, Bartosz Grzybowski, Tarik Baytekin, and Bilge Baytekin for helpful discussions. This project has received funding from the European Unions Horizon 2020 research and innovation programme under the Marie Skłodowska-Curie Grant Agreement No. 754411.
[1] D. J. Lacks and M. R. Sankaran, J. Phys. D: Appl. Phys. 44, 453001 (2011).

[2] D. J. Lacks and T. Shinbrot, Nat. Rev. Chem. 3, 465 (2019).

[3] T. Steinpilz, K. Joeris, F. Jungmann, D. Wolf, L. Brendel, J. Teiser, T. Shinbrot, and G. Wurm, Nat. Phys. 16, 225 (2020).

[4] S. J. Desch and J. N. Cuzzi, Icarus 143, 87 (2000).

[5] G. Wurm, L. Schmidt, T. Steinpilz, L. Boden, and J. Teiser, Icarus 331, 103 (2019).

[6] D. L. Schrader, K. Nagashima, S. R. Waitukaitis, J. Davidson, T. J. McCoy, H. C. Connolly Jr., and D. S. Lauretta, Geochim. Cosmochim. Acta 223, 405 (2018).

[7] P. Berdeklis and R. List, J. Atmos. Sci. 58, 2751 (2001).

[8] H. T. Baytekin, B. Baytekin, T. M. Hermans, B. Kowalczyk, and B. A. Grzybowski, Science 341, 1368 (2013).

[9] T. Abbasi and S. A. Abbasi, J. Hazard. Mat. 140, 7 (2007).

[10] M. Kanik, O. Aktas, H. S. Sen, E. Durgun, and M. Bayindir, ACS Nano 8, 9311 (2014).

[11] U. G. Musa, S. D. Cezan, B. Baytekin, and H. T. Baytekin, Sci. Rep. 8, 2472 (2018).

[12] Z. L. Wang, ACS Nano 7, 9533 (2013).

[13] W. Hu, L. Xie, and X. Zheng, Appl. Phys. Lett. 101, 114107 (2012).

[14] S. R. Waitukaitis and H. M. Jaeger, Rev. Sci. Instrum. 84, 025104 (2013).

[15] S. R. Waitukaitis, V. Lee, J. M. Pierson, S. L. Forman, and H. M. Jaeger, Phys. Rev. Lett. 112, 218001 (2014).

[16] A. L. Collins, C. G. Camara, E. V. Van Cleve, and S. J. Putterman, Rev. Sci. Instrum. 89, 013901 (2018).

[17] V. Lee, N. M. James, S. R. Waitukaitis, and H. M. Jaeger, Phys. Rev. Materials 2, 035602 (2018).
[18] I. A. Harris, M. X. Lim, and H. M. Jaeger, Phys. Rev. Materials 3, 085603 (2019).

[19] J. Lowell and W. S. Truscott, J. Phys. D: Appl. Phys. 19, 1273 (1986).

[20] K. M. Forward, D. J. Lacks, and R. M. Sankaran, Geophys. Res. Lett. 36, L13201 (2009).

[21] J. Lowell and W. S. Truscott, J. Phys. D: Appl. Phys. 19, 1281 (1986).

[22] D. J. Lacks, N. Duff, and S. K. Kumar, Phys. Rev. Lett. 100, 188305 (2008).

[23] D. J. Lacks and A. Levandovsky, J. Electrost. 65, 107 (2007).

[24] N. Duff and D. J. Lacks, J. Electrost. 66, 51 (2008).

[25] D. J. Lacks and R. M. Sankaran, Part. Sci. Technol. 34, 55 (2016).

[26] T. Shinbrot, M. Rutala, and H. Herrmann, Phys. Rev. E 96, 032912 (2017).

[27] T. Siu, J. Cotton, G. Mattson, and T. Shinbrot, Phys. Rev. E 89, 052208 (2014).

[28] J. Kolehmainen, A. Ozel, Y. Gu, T. Shinbrot, and S. Sundaresan, Phys. Rev. Lett. 121, 124503 (2018).

[29] R. Yoshimatsu, N. A. M. Araújo, G. Wurm, H. J. Herrmann, and T. Shinbrot, Sci. Rep. 7, 39996 (2016).

[30] R. Yoshimatsu, N. A. M. Araújo, T. Shinbrot, and H. J. Herrmann, Soft Mater. 12, 6261 (2016).

[31] M. Sow, D. J. Lacks, and M. R. Sankaran, J. Appl. Phys. 112, 084909 (2012).

[32] M. Sow, R. Widenor, A. Kumar, S. Lee, D. Lacks, and R. Sankaran, Ang. Chem. Int. Ed. 51, 2695 (2012).

[33] M. Sow, D. J. Lacks, and R. M. Sankaran, J. Electrost. 71, 396 (2013). 
[34] M. M. Apodaca, P. J. Wesson, K. J. M. Bishop, M. A. Ratner, and B. A. Grzybowski, Angew. Chem. Int. Ed. 49, 946 (2010).

[35] H. T. Baytekin, A. Z. Patashinski, M. Branicki, B. Baytekin, S. Soh, and B. A. Grzybowski, Science 333, 308 (2011).

[36] See Supplemental Material at http://link.aps.org/supplemental/ 10.1103/PhysRevMaterials.4.082602 for the theory and simulations, a discussion on multiple length scales, as well as a reconsideration of previous experimental results.

[37] J. Haeberle, A. Schella, M. Sperl, M. Schrter, and P. Born, Soft Matter 14, 4987 (2018).

[38] A. E. Wang, P. S. Gil, M. Holonga, Z. Yavuz, H. T. Baytekin, R. M. Sankaran and D. J.L acks, Phys. Rev. Materials 1, 035605 (2017).
[39] L. Xie, N. Bao, Y. Jiang, and J. Zhou, AIP Adv. 6, 035117 (2016).

[40] H. Yu, L. Mu, and L. Xie, J. Electrost. 90, 113 (2017).

[41] K. Xu, P. Cao, and J. R. Heath, Science 329, 1188 (2010).

[42] T. Burgo, F. Galembeck, and G. Pollack, J. Electrost. 80, 30 (2016).

[43] L. B. da Silveira Balestrin, D. Del Duque, D. S. da Silva, and P. Galembeck, Faraday Discuss. 170, 369 (2014).

[44] H. Brune, Surf. Sci. Rep. 31, 121 (1998).

[45] K. K. Varanasi, M. Hsu, N. Bhate, W. Yang, and T. Deng, Appl. Phys. Lett. 95, 094101 (2009).

[46] B. Lü, G. A. Almyras, V. Gervilla, J. E. Greene, and K. Sarakinos, Phys. Rev. Materials 2, 063401 (2018).

[47] J. F. Kok and D. J. Lacks, Phys. Rev. E 79, 051304 (2009). 